\title{
Systemic hypertension followed by insidious stroke in a 12-year-old boy with childhood neurofibromatosis type 1 presenting with renal and cerebral artery vasculopathy
}

\author{
Meng-Luen Lee ${ }^{1,2}$, Tung-Ming Chang ${ }^{3}$, Rei-Cheng Yang ${ }^{3}$, Albert D. Yang ${ }^{4}$, Ming Chen ${ }^{5,6}$ \\ Divisions of ${ }^{1}$ Pediatric Cardiology and ${ }^{3}$ Pediatric Neurology, Department of Pediatrics and Departments of ${ }^{4}$ Medical Imaging \\ and ${ }^{5}$ Medical Genetics, Changhua Christian Children's Hospital, Changhua; ${ }^{2}$ School of Medicine, Kaohsiung Medical \\ University, Kaohsiung; ${ }^{6}$ Department of Medical Genetics, College of Medicine, National Taiwan University Hospital, \\ Taipei, Taiwan. E-mail: ferdielee@yahoo.com \\ Received: 10th April 2018, Received: 2nd May 2018, Accepted: 17th May 2018
}

SUMMARY: Lee ML, Chang TM, Yang RC, Yang AD, Chen M. Systemic hypertension followed by insidious stroke in a 12-year-old boy with childhood neurofibromatosis type 1 presenting with renal and cerebral artery vasculopathy. Turk J Pediatr 2019; 61: 629-634.

Neurofibromatosis type 1 (NF1)-associated vasculopathy is usually diagnosed decades after the clinical diagnosis of NF1. Childhood NF1-associated renal artery vasculopathy or moyamoya-like brain vasculopathy could be clinically silent for a long time. We report a 12-year-old boy who had systemic hypertension found incidentally at a routine check-up. Physical examination showed caféau-lait spots and strong radial pulses. Abdominal computerized tomography angiography showed severe right ostial renal artery stenosis. Genomic study showed a heterozygous mutation c.5902C > T (p.R1968*) and two heterozygous single nucleotide polymorphisms (NCBI: SNP rs18011052 and rs2905876) of NF1 gene. After endovascular revascularization for renovascular hypertension caused by renal artery stenosis, including percutaneous transluminal renal angioplasty and stent implantation, blood pressure dropped effectively from $205 / 143 \mathrm{mmHg}$ to $130 / 90 \mathrm{mmHg}$. Supine renin level dropped from $87.2 \mathrm{pg} /$ $\mathrm{mL}$ to $47.9 \mathrm{pg} / \mathrm{mL}$. Unfortunately, right hemiplegia, transient visual loss with blind spots (scotomas), and clumsiness of extremities emerged insidiously 3.5 months later. Brain magnetic resonance imaging and magnetic resonance angiography showed ischemic infarction involving the watershed area of the anterior and middle cerebral arteries, indicating presence of moyamoya-like brain vasculopathy. A dilemma is that a significant decrease of blood pressure after endovascular revascularization for renal artery stenosis may have potentially unmask the moyamoya-like brain vasculopathy in this patient. Vasculopathy could be heralding childhood NF1 in the young patients without full-fledged clinical features. Endovascular revascularization for renal artery stenosis could be a double-edge sword in childhood NF1 presenting with concomitant renal and cerebral artery vasculopathy.

Key words: neurofibromatosis type 1, vasculopathy, percutaneous transluminal renal angioplasty, stent implantation, moyamoya syndrome.

Neurofibromatosis type 1 (NF1; OMIM $\# 162200)$ is an inherited multisystem disorder caused by dominant loss-offunction mutations of the tumor-suppressor gene neurofibromin 1 (NF1; OMIM \#613113) which is located at $17 \mathrm{q} 11.2 .^{1}$ A diagnosis of NF1 can be made if two of the following criteria are met, including café-au-lait spots, intertriginous freckling, dermal neurofibroma, optic glioma, Lisch nodules, skeletal dysplasia, and a positive family history. ${ }^{2}$ We report a case who presented clinically with silent renal and cerebral arterial vasculopathy, illustrating the caveats in the diagnosis and management of childhood NF1. 


\section{Case Report}

A 12-year-old boy was referred to our tertiary medical center with a chief complaint of systemic hypertension (190/98 $\mathrm{mmHg})$ found incidentally at a routine check-up of blood pressure at a local clinic. At referral, he remained asymptomatic. Café-au-lait spots were discernible (Fig. 1a). Under the presumed diagnosis of NF1, abdominal computerized tomography angiography was performed and established a severe right ostial renal artery stenosis (RAS) (Fig. 1b, left panel), total occlusion of the celiac trunk, and moderate stenosis of the superior mesenteric artery (SMA) (Fig. 1b, right panel). On admission, he weighed $46 \mathrm{~kg}$ and $156 \mathrm{~cm}$ in height. Blood pressure was 190/98 $\mathrm{mmHg}$, heart rate $108 / \mathrm{min}$, and respiratory rate $22 /$ min. Bilateral radial/femoral pulses were bounding. There were no abdominal and carotid bruits. Electrocardiogram showed left ventricular hypertrophy by voltage. Erythrocyte sedimentation rate was $11 \mathrm{~mm} /$ hr. Genomic study showed a heterozygous mutation c.5902C>T (p.R19689*) and two heterozygous single nucleotide polymorphisms (NCBI: SNP rs18011052 and rs2905876) of NF1 gene (Fig. 1c). By cardiac catheterization, aortic pressure was 205/143 $\mathrm{mmHg}$. Angiography of the right renal artery confirmed ostial RAS $(2.24 \mathrm{~mm}$; stenosis ratio of $59 \%$ ). Percutaneous transluminal renal angioplasty (PTRA) was performed after informed consent from his parents. We used a coronary balloon catheter of $4.5 \mathrm{~mm}$ x $15 \mathrm{~mm}$ (Sprinter Legend, Medtronic, Galway, Ireland) to perform PTRA, with a distended pressure increased gradationally from $6 \mathrm{~atm}$ to $14 \mathrm{~atm}$ (a maximum diameter of $4.55 \mathrm{~mm}$ ). Stenosis ratio improved from $59 \%$ to $10 \%$. Supine renin level dropped from $29.2 \mathrm{pg} / \mathrm{mL}$ to 20.4 $\mathrm{pg} / \mathrm{mL}$ (normal range: $<3.6-20.1 \mathrm{pg} / \mathrm{mL}$ ). However, blood pressure fluctuated around $150-170 / 90-110 \mathrm{mmHg}$ in the following three weeks. Supine renin level increased from 20.4 $\mathrm{pg} / \mathrm{mL}$ to $87.2 \mathrm{pg} / \mathrm{mL}$. Under the impression of restenosis of the right renal artery, he was admitted for repeated PTRA. Aortic pressure was $183 / 106 \mathrm{mmHg}$ on cardiac catheterization. Angiography showed a long-segment right RAS $(0.42 \mathrm{~mm}$; stenosis ratio of $87 \%)$ (Fig. $1 \mathrm{~d}$, left panel). At first, we used two sets of coronary balloon catheter $(3.5 \mathrm{~mm} \times 20 \mathrm{~mm}$, Sprinter Legend, Medtronic, Galway, Ireland; $5.00 \mathrm{~mm}$ x 12 mm, NC TREK, Abbott Vascular, Diegem, Belgium) to dilate this long-segment right RAS stepwise from the main trunk to the ostium of the right renal artery, with no success. Afterward, we used a longer coronary balloon catheter $(3.5 \mathrm{~mm} \times 30 \mathrm{~mm}$, Sprinter Legend, Medtronic, Galway, Ireland) to perform highpressure PTRA, with a distended pressure maintained at 14 atm and for 2 minutes. However, the restenosis remained refractory to high-pressure PTRA. A stent was implanted to prevent total occlusion of the right renal artery $(4.0 \mathrm{~mm} \times 38 \mathrm{~mm}$, Abbott Coronary Stent, Abbott Vascular, Diegem, Belgium). Post-intervention angiography showed patent flow from the abdominal aorta through the right renal artery to the right kidney (Fig. 1d, right panel). Blood pressure dropped from $183 / 106 \mathrm{mmHg}$ to $130 / 90 \mathrm{mmHg}$. Supine renin level dropped from $87.2 \mathrm{pg} / \mathrm{mL}$ to $47.9 \mathrm{pg} / \mathrm{mL}$. Aspirin was prescribed for anticoagulation. Clinical symptoms and signs of transient ischemic attack (TIA), including right hemiplegia, transient visual loss with blind spots (scotomas), and clumsiness of extremities, emerged insidiously 3.5 months after the intervention. Brain magnetic resonance imaging (MRI) showed ischemic infarction involving the deep watershed zone of the anterior and middle cerebral arteries, including T2-weighted and fluid-attenuated inversion recovery images (Fig. 2a), diffusion weighted imaging with apparent diffusion coefficient (Fig. 2b), and T1-weighted images with gadolinium enhancement (Fig. 2c). Brain magnetic resonance angiography (MRA) showed stenoses of intracranial carotid arteries and bilateral middle cerebral arteries (Fig. 2d), indicating moyamoya syndrome (MMS) associated with NF1. His intelligence quotation score was 71 (normal range: 90110), which was assessed by the Wechsler Intelligence Scale for Children. He was later lost in the follow-up.

\section{Discussion}

This case carries five points to be highlighted. First, the patient presented with only one criterion of NF1, namely, cutaneous café-aulait spots. However, genomic study showed a 

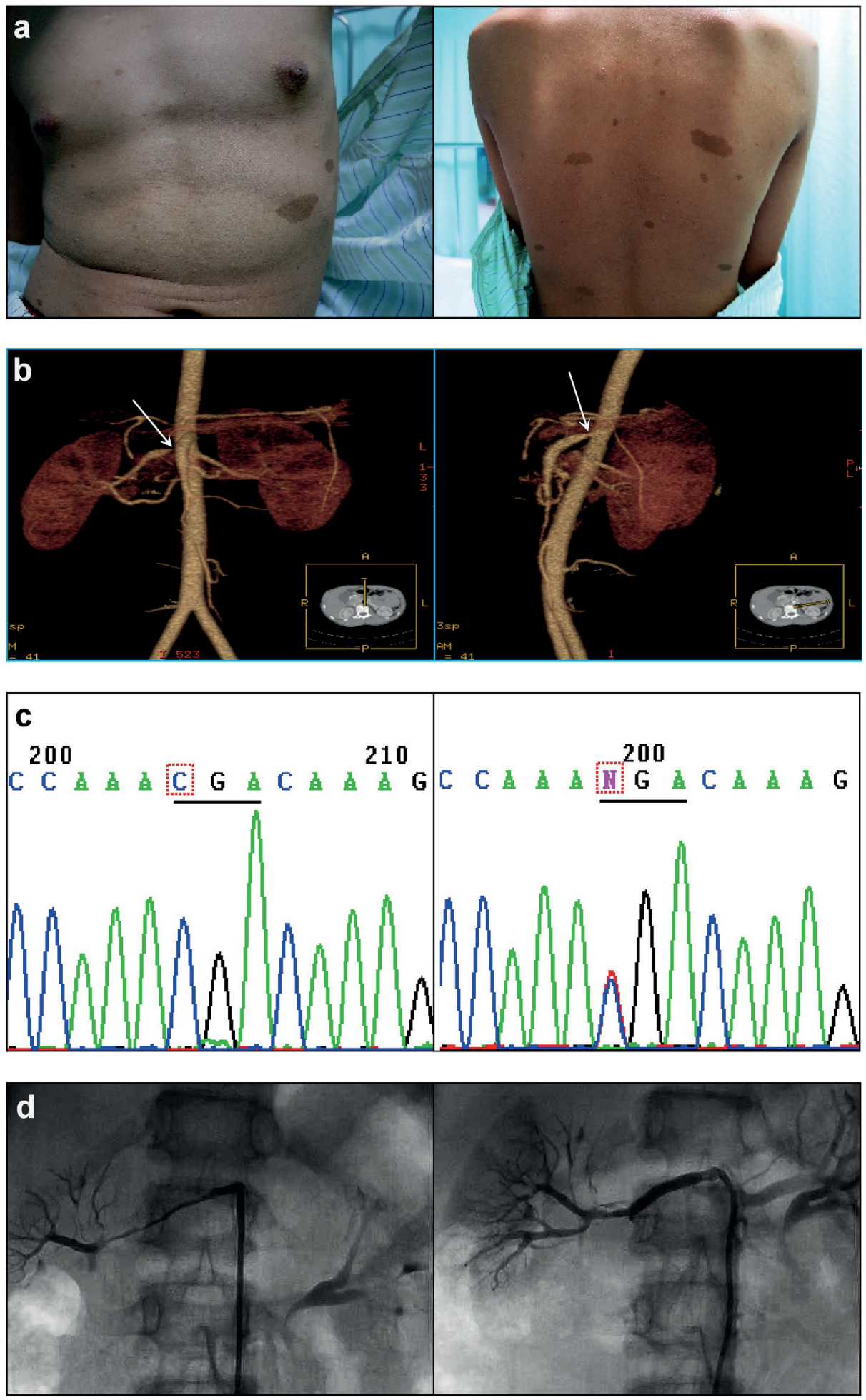

Fig. 1. (a) Café-au-lait spots were noted over abdomen (left panel) and back trunk (right panel). (b) Abdominal computerized tomography angiography showed severe right ostial renal artery stenosis (left panel) and stenosis of the superior mesenteric artery (right panel). (c) Genomic study showed a heterozygous mutation c.5902C>T (p.R1968*) and two heterozygous single nucleotide polymorphisms (NCBI: SNP rs18011052 and rs2905876) of the NF1 gene. (d) Right renal arteriography showed a long-segment restenosis of the right renal artery after percutaneous transluminal renal angioplasty (left panel), and patent blood flow through the right renal artery after stent implantation (right panel). 

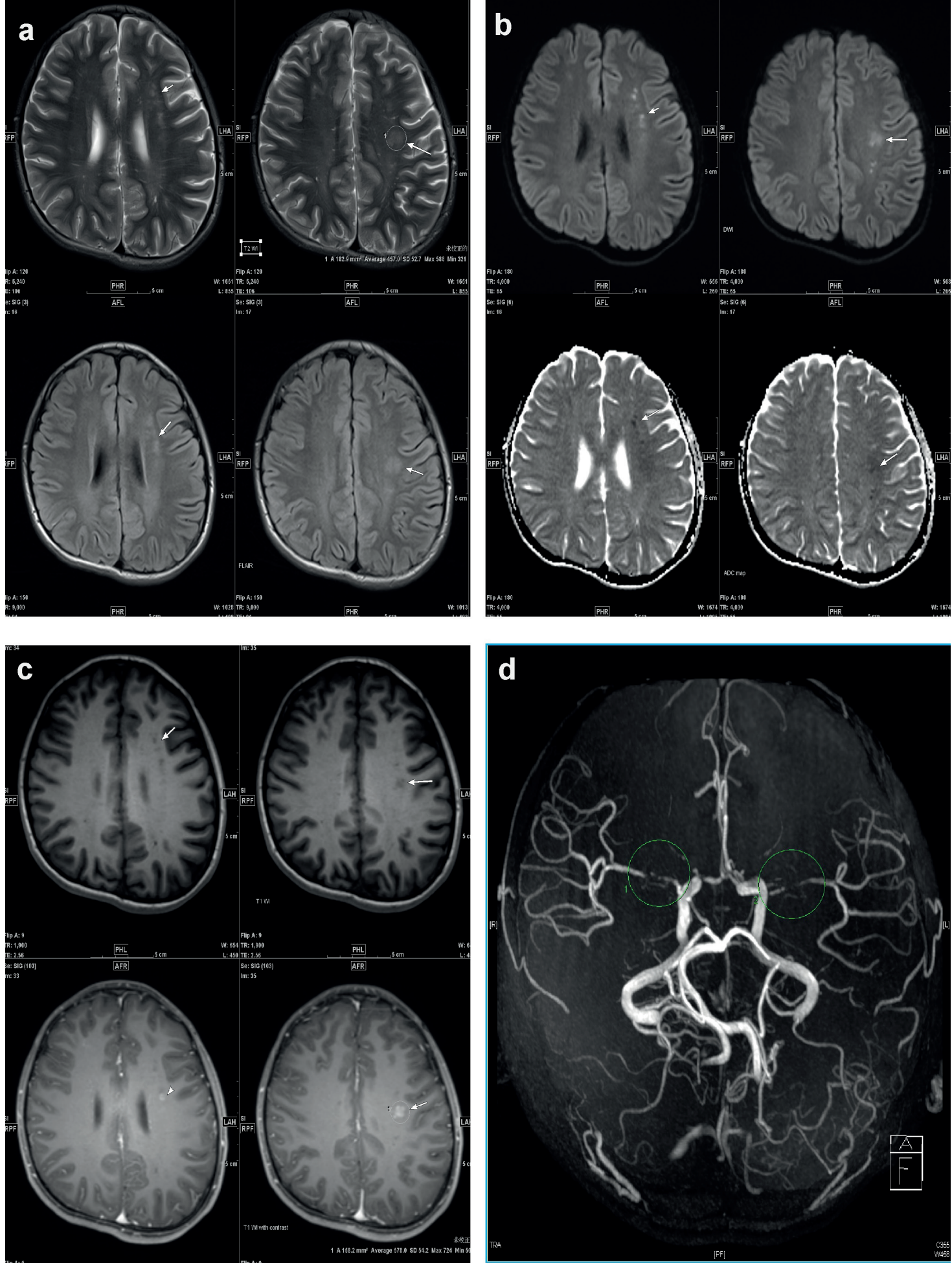

Fig. 2. Brain magnetic resonance imaging showed ischemic infarction involving the watershed zone of the anterior and middle cerebral arteries (arrows), including (a) T2-weighted images (upper two panels) and fluid-attenuated inversion recovery (FLAIR) images (lower two panels), (b) diffusion weighted imaging (DWI; upper two panels) and apparent diffusion coefficient (ADC; lower two panels) mapping, and (c) T1weighted images without gadolinium contrast enhancement (upper two panels), and T1-weighted images with gadolinium contrast enhancement (lower two panels). (d) Brain magnetic resonance angiography showed stenoses involving bilateral middle cerebral arteries (circles). 
heterozygous mutation c.5902C > T (p.R1968*) and two heterozygous single nucleotide polymorphisms (NCBI: SNP rs18011052 and rs2905876) in the NF1 gene. DeBella et al. ${ }^{2}$ reported that approximately $46 \%$ of sporadic cases fail to meet the NIH Diagnostic Criteria by 1 year of age whereas $97 \%$ meet the criteria by 8 years old, and all do so by 20 years old, and they proposed that a modification of the diagnostic criteria for NF1 in children. Given that NF1 mutation could be detected in $97 \%$ of 565 patients with NF1, ${ }^{1}$ NF1 mutation analysis may be suggested in young children without full-fledged features of NF1.

Second, NF1 vasculopathy was coined to describe a pleiotropic array of vascular abnormalities identified in patients with NF1. ${ }^{3}$ In four large series of childhood NF1, the prevalence of cerebral artery vasculopathy was $2.5 \%-6.4 \%$, and that of MMS was $0.6 \%$ $4.9 \%{ }^{4-7}$ Considering relatively low incidence of cerebral artery vasculopathy and MMS in childhood NF1, clinical guidelines did not advocate neuroimagings in the asymptomatic patients as routine examinations. ${ }^{8}$ Oderich et al. ${ }^{9}$ reported that the incidence of vasculopathy of renal artery, carotid-vertebral-cerebral artery, and aorta were $41 \%, 19 \%$, and $12 \%$, respectively. In this regard, routine check-up of blood pressure is warranted in patients with suspected NF1, and systemic hypertension can be served as a harbinger of NF1 that is most liable to incur renal artery vasculopathy in terms of RAS. Overall, a diagnosis of NF1 and NF1 vasculopathy was made at $11 \pm$ 10 years and $38 \pm 16$ years, respectively, ${ }^{9}$ which implies that vasculopathy emerged after a lapse of two to three decades and the incidence of vasculopathy will increase with time. Thus, vasculopathy is a hallmark not only heralding childhood NF1 in the young population without full-fledged features, but also implicating excess deaths in patients $<30$ years of age. ${ }^{10}$ It is foresighted to include vasculopathy as a diagnostic clue to facilitate an early diagnosis of childhood NF1, especially in those cases presenting with renovascular hypertension, TIA or stroke (cerebral artery vasculopathy), differential hypertension of the upper limbs (thoracic aorta vasculopathy), intestinal gangrene (SMA or celiac trunk vasculopathy), and disabling claudication (aorto-iliac artery vasculopathy).

Third, a thorough understanding of the pathogenesis of NF1 vasculopathy may clarify its progressive or relapsing nature. NF1 vasculopathy is assumed to be a developmental lesion, with a genetic predisposition to progression. ${ }^{11}$ It is given that neurofibromin, encoded by the NF1 gene, serves as a GTPase activating protein that accelerates conversion of active Ras-GTP to inactive Ras-GDP. ${ }^{11}$ The mutation of the NF1 gene results in loss of neurofibromin expression in the endothelial cells, accumulation of RasGTP, and a perturbation of vasculogenesis. ${ }^{11}$ Intriguingly, the alteration of the morphogenic phenotype of NF1 vasculopathy appears to be quite plastic, for the acquisition of an additional mutation or epigenetic silencing of the remaining copy of NF1 gene may trigger Ras activation and render autonomous proliferation and abnormal formation in the post-developmental quiescent vasculature. ${ }^{11}$ That is why NF1 vasculopathy may display a plethora of morphology, including aneurysm, stenosis, arteriovenous malformation, and moyamoya. ${ }^{9,11}$

Fourth, antihypertensive agents were usually palliative, but not curative, for renovascular hypertension due to RAS in childhood NF1. Because of the progressive nature of childhood RAS, repeated PTRA has been advocated as an initial treatment of choice, disregarding its underlying disease. ${ }^{12}$ Stent implantation should be reserved as a bailout procedure for childhood RAS, if results are suboptimal or dissection occurs after PTRA. ${ }^{13}$

Finally, moyamoya disease is referred to the occlusion of bilateral arteries of the circle of Willis of unknown origin, and MMS is defined as an underlying disease having similar pathognomonic arteriographic finding of a puff of smoke (moyamoya). Surgical revascularization is safe and effective in preventing further ischemic and neurological damage in children with NF1-associated MMS. ${ }^{14}$ However, it can be a double-edge sword for childhood MMS because it may also cause postoperative neurological deterioration due to cerebral ischemia (watershed shift) or cerebral hyperperfusion syndrome. ${ }^{15} \mathrm{~A}$ significant decrease of blood pressure by 
intervention may lead to ischemic infarction by a vascular steal phenomenon of the watershed zones and cause TIA or cerebrovascular stroke in the present patient.

In conclusion, renal and cerebral vasculopathy in NF1 can be silent for a long time. The pediatrician's role is crucial for a timely diagnosis of these complicated but treatable conditions, as measurement of the blood pressure and examination of the skin during routine pediatric visits provide the diagnostic clues.

\section{REFERENCES}

1. Sabbagh A, Pasmant E, Imbard A, et al. NF1 molecular characterization and neurofibromatosis type I genotype-phenotype correlation: The French experience. Hum Mutat 2013; 34: 1510-1518.

2. DeBella K, Szudek J, Friedman JM. Use of the national institutes of health criteria for diagnosis of neurofibromatosis 1 in children. Pediatrics 2000; 105(3 Pt 1): 608-614.

3. Hamilton SJ, Friedman JM. Insights into the pathogenesis of neurofibromatosis 1 vasculopathy. Clin Genet 2000; 58: 341-344.

4. Rosser TL, Vezina G, Packer RJ. Cerebrovascular abnormalities in a population of children with neurofibromatosis type 1 . Neurology 2005; 64: 553555.

5. Ghosh PS, Rothner AD, Emch TM, Friedman NR, Moodley M. Cerebral vasculopathy in children with neurofibromatosis type 1 . J Child Neurol 2013; 28: 95-101.

6. Cairns AG, North KN. Cerebrovascular dysplasia in neurofibromatosis type 1. J Neurol Neurosurg Psychiatry 2008; 79: 1165-1170.
7. Rea D, Brandsema JF, Armstrong D, et al. Cerebral arteriopathy in children with neurofibromatosis type 1. Pediatrics 2009; 124: e476-e483.

8. Williams VC, Lucas J, Babcock MA, Gutmann DH, Korf B, Maria BL. Neurofibromatosis type 1 revisited. Pediatrics 2009; 123: 124-133.

9. Oderich GS, Sullivan TM, Bower TC, et al. Vascular abnormalities in patients with neurofibromatosis syndrome type I: Clinical spectrum, management, and results. J Vasc Surg 2007; 46: 475-484.

10. Rasmussen SA, Yang Q, Friedman JM. Mortality in neurofibromatosis 1: An analysis using U.S. death certificates. Am J Hum Genet 2001; 68: 1110-1118.

11. Bajaj A, Li QF, Zheng Q, Pumiglia K. Loss of NF1 expression in human endothelial cells promotes autonomous proliferation and altered vascular morphogenesis. PLoS One 2012; 7: e49222.

12. Srinivasan A, Krishnamurthy G, Fontalvo-Herazo L, et al. Angioplasty for renal artery stenosis in pediatric patients: An 11-year retrospective experience. J Vasc Interv Radiol 2010; 21: 1672-1680.

13. Tyagi S, Kaul UA, Arora R. Endovascular stenting for unsuccessful angioplasty of the aorta in aortoarteritis. Cardiovasc Intervent Radiol 1999; 22: 452-456.

14. Koss M, Scott RM, Irons MB, Smith ER, Ullrich NJ. Moyamoya syndrome associated with neurofibromatosis Type 1: Perioperative and longterm outcome after surgical revascularization. J Neurosurg Pediatr 2013; 11: 417-425.

15. Hayashi T, Shirane R, Fujimura M, Tominaga T. Postoperative neurological deterioration in pediatric moyamoya disease: Watershed shift and hyperperfusion. J Neurosurg Pediatr 2010; 6: 73-81. 